Running head: REJECTION SENSITIVITY AND SOCIAL ANXIETY

\author{
Rejection Sensitivity and the Development of Social Anxiety \\ Symptoms during Adolescence: A Five-Year Longitudinal Study
}

Melanie J. Zimmer-Gembeck, Alex A. Gardner, Tanya Hawes, Mitchell R. Masters, Allison M. Waters, Lara J. Farrell

School of Applied Psychology, Griffith University, Gold Coast, QLD, Australia

\begin{abstract}
Acknowledgements
This research was funded by Australian Research Council Discovery Grants (DP130101868 and DP170102547). We thank Drew Nesdale, Geraldine Downey and Haley Webb for advice during the early stages of the larger project from which these data were drawn.
\end{abstract}




\begin{abstract}
Rejection sensitivity is a bias towards expecting rejection that can result from negative social experiences and degrade emotional adjustment. In this study, rejection sensitivity was expected to predict patterns of adolescent social anxiety over five years when considered alongside other known or expected risk and protective factors: peer rejection (peer-reported), emotion dysregulation, self-worth, temperament (parent-reported), female gender, and grade. Participants were 377 Australian students (45\% boys; 79\% white, 15\% Asian) aged 10 to 13 years $(M=12.0, S D=0.90)$ and their parents $(84 \%)$ who completed seven repeated surveys across five years. In an unconditional latent growth model, social anxiety symptoms had a significant quadratic pattern of growth, with symptoms increasing about mid-way into the study when adolescents were age 14, on average. In a model with all predictors, rejection sensitivity was uniquely associated with a higher intercept and a more pronounced quadratic growth pattern of social anxiety symptoms. Other predictors of growth in symptoms were the temperamental trait of negativity affectivity and emotion dysregulation; negative affectivity was associated with a higher intercept and a more pronounced quadratic pattern, and emotion dysregulation was associated with a higher intercept and a less pronounced quadratic pattern. Gender was associated with the intercept, with girls higher in symptoms than boys.
\end{abstract}

Keywords: rejection sensitivity, social anxiety, emotion regulation, peer influence, temperament 


\section{Rejection Sensitivity and the Development of Social Anxiety Symptoms during Adolescence: A Five-Year Longitudinal Study}

The adolescent years have been identified as a high-risk time for the escalation of social anxiety symptoms and the onset of disorder, with the median age of onset typically reported to occur at or before age 15 years (Nelemans et al., 2019; Rapee et al., 2019). With the goal of prevention and intervention of adolescent social anxiety in mind, theorists have drawn from developmental and clinical perspectives to identify early socio-environmental risk factors for social anxiety, including exposure to violence; inconsistent, coercive, or rejecting parenting practices; and rejection, victimization, or isolation from peers (e.g., Clear et al., 2020; Spence \& Rapee, 2016). What the above social-environmental risks have in common is their potentially negative impact on children's social-cognitive beliefs about the behaviors they can expect from other people (Bowlby, 1969).

Negative social-cognitive beliefs about others include attentional biases to the possibility of judgement by others (Spence \& Rapee, 2016; Weeks et al., 2009) and biased appraisals or interpretation of others' behaviors as more rejecting and hostile (Downey et al., 1998a; Gardner \& Zimmer-Gembeck, 2018). One form of biased appraisal of others' behaviors is rejection sensitivity, defined as a cognitive-affective processing system that involves anxiously expecting, readily perceiving, and defensively reacting in situations where rejection or exclusion is possible or threatened (Downey \& Feldman, 1996). Rejection sensitivity theory argues that it develops

through environmental experiences (Downey et al., 1999) and research finds that it is elevated among adolescents with a greater history of rejection and victimization by parents or peers (Chango et al., 2012; Rowe et al., 2015). Thus, rejection sensitivity may be a proximal mechanism that explains why aversive social experiences coalesce in emotional maladjustment, 
including being a risk for the development of social anxiety symptoms (Abela \& Hankin, 2009; Zimmer-Gembeck et al., 2014). However, there has been limited empirical examination of the role of rejection sensitivity in the development of adolescents' social anxiety symptoms. The purpose of this study was to investigate the risk conferred by elevated rejection sensitivity at the transition to adolescence for development of social anxiety symptoms across the following five years, while simultaneously accounting for peer rejection and other known or possible personal risk factors for social anxiety symptom development.

\section{Peer Rejection, Rejection Sensitivity and Social Anxiety}

Peer relationships play an important role in the lives of adolescents, impacting on their sense of acceptance and belonging (Laursen \& Collins, 2009; Troop-Gordon, 2017). Yet, peer relationships are not always accepting and inclusive. In fact, research indicates that between 10 to $16 \%$ of adolescents report peer rejection or victimization (Duffy et al., 2020), and such rejection has been associated with rejection sensitivity (Gómez-Ortiz et al., 2017; ZimmerGembeck et al., 2013). Furthermore, rejection sensitivity has been described as a mechanism that explains why rejection experiences are associated with maladjustment in multiple domains (Downey \& Feldman, 1996; Downey et al., 1998a), and rejection sensitivity does seem to have far-reaching negative implications for psychosocial adjustment (see Duffy et al., 2020 or Zimmer-Gembeck, 2016 for reviews). Most relevant to the current study, rejection sensitivity has been associated with more social anxiety among adolescents (Bowker et al., 2011; Ding et al., 2020), and rejection sensitivity has been identified as a predictor of increased symptoms across a short period of time during late adolescence and young adulthood (Gardner et al., 2020).

It may not be surprising that previous research has found positive concurrent (and even short-term prospective) associations between rejection sensitivity and social anxiety, as they do 
share some conceptual similarity. In particular, rejection sensitivity and social anxiety each refer to emotions in situations of perceived or actual interpersonal threat or challenge. However, there are also important distinctions between them. First, focusing on rejection sensitivity narrows the focus from general self-presentational concerns of social anxiety to a specific focus on expectations and anxious concerns about rejection and exclusion by others. This more explicitly ties risk for the development of social anxiety to early socio-environmental experiences and expectations for others' behaviors, areas that deserve more attention in interventions and treatments. Second, social anxiety symptoms and rejection sensitivity are differentiated by an emphasis on responses versus expectations. Social anxiety symptoms identify how individuals themselves respond or react to social interactions and events, whereas rejection sensitivity focuses on expectations of others' responses. Third, like other attentional, perceptual, and socialinformation processing biases that have been shown to be associated with social anxiety and share some conceptual similarity (e.g., attentional vigilance to threat), there is a great deal of clinical theory and research that identifies biases as risk factors for the development of symptoms and disorder, not part of the disorder itself (Rapee et al., 2019; Spence \& Rapee, 2016). We argue here that this is especially true in longitudinal studies when a bias is measured prior to the known timing of the escalation or onset of disorder.

Overall, rejection sensitivity may be a particularly powerful and unique risk factor for social anxiety symptom increase during adolescence. Not only does it imply interference in fulfilling the need for belonginess and acceptance by others, which can cause worry and distress about social relationships and impact negatively on emotional health (Baumeister \& Leary, 1995), it also captures biased views of the quality of social interactions and relationships that may be generally positive. Thus, even when relationships may appear generally positive by an 
external standard, individuals high in rejection sensitivity may not experience the benefits, making them even more concerned and worried about their social interactions over time (Ayduk et al., 1999; Downey et al., 1998a). Thus, a wider and wider set of social events may be perceived as possibilities for rejection and exclusion (e.g., simply eating lunch in front of others, speaking in class), eventually culminating in social anxiety symptoms and, possibly, disorder. Furthermore, rejection sensitivity may lead to self-blame (Hankin \& Abramson, 2001; ZimmerGembeck et al., 2016) and interfere with positive responses (e.g., positive thinking, seeking support) that could repair negative emotions following (real or perceived) poor social interactions, instead being linked to social withdrawal, hostile reactions, or seeking retribution for either real or perceived rejection (Williams, 2001; Zimmer-Gembeck et al, 2016). As negative responses become more chronic and interfere with existing relationship development, they can involve a cycle of perceived poor social interactions, more worries, and maladaptive responses to social situations that, together, sustain social isolation, limit remedial experiences (e.g., acceptance by others), and result in growth in social anxiety symptoms.

\section{Emotion Dysregulation and Social Anxiety}

In the present study, it was our aim to consider the unique risk of rejection sensitivity for social anxiety development independent from the effects of other established risk factors. Emotion dysregulation was one of these other risk factors, given it has been found to be positively related to concurrent and increasing social anxiety symptoms (Carthy et al., 2010; Gross \& Jazaieri, 2014). For example, in a 3-year study from the initial waves of the data analyzed here, emotion dysregulation was associated with more elevated social anxiety symptoms about one year later, and social anxiety, in turn, predicted worsening dysregulation one year later (Masters et al., 2019). However, this study focused only on bidirectional 
associations between each wave rather than growth in social anxiety symptoms across adolescence, and considered only emotion regulation and psychological maladjustment, not rejection sensitivity or other potential risks for social anxiety development. More generally, there has been an upsurge in research on emotion regulation and dysregulation as risk markers and/or protective factors in the ontogenesis of social anxiety symptoms and disorder (Carthy et al., 2010; Gross \& Jazaieri, 2014). Influenced by theories of stress and coping, emotion regulation has been described as a transdiagnostic risk for many disorders that are amenable to intervention efforts (Compas et al., 2017; Dryman \& Heimberg, 2018), and it is possible that emotion dysregulation positively covaries with rejection sensitivity in adolescents, as has been found in university students (Gardner et al., 2020).

Emotion dysregulation encompasses a multi-faceted set of processes, such as heightened emotional reactivity, prolonged duration and failure to modulate emotions, and less awareness, efficacy or flexibility in recognizing and responding to this reaction; any of which may interfere with daily functioning and goal-directed activity (Adrian et al., 2019; Thompson, 2019). In adolescence, youth continue to learn, through socializing agents, how to adaptively regulate emotions, and failures of regulation can give rise to chronic negative affect, increased symptomology, and for some, the development of emotional disorders (Mathews et al., 2014; Rapee et al., 2019; Thompson, 2019). Evidence exists that, compared to non-anxious youth, youth with a diagnosis of an anxiety disorder (Suveg \& Zeman, 2004), including social anxiety disorder (Sackl-Pammer et al., 2019), demonstrate greater emotional intensity and dysregulated emotional expression, feel less confident in regulating emotions, and report using more maladaptive strategies (e.g., rumination and avoidance) and fewer adaptive strategies (e.g., cognitive reappraisal). Similar associations have been found in community samples of 
adolescents (Golombek et al., 2019; Mathews et al., 2014).

\section{Perceived Self-worth and Social Anxiety}

Just as negative socio-environmental experiences of rejection, victimization, and exclusion has been found to be linked to elevated rejection sensitivity, they can also result in poor self-worth, which could, in turn, be a proximal link to emotional problems such as social anxiety (Lopez \& DuBois, 2010). Adolescents with more social anxiety symptoms report poorer self-esteem and poorer perceptions of their self-worth (Bowker et al., 2011; Gómez-Ortiz et al., 2017; Weeks et al., 2009). Such associations suggest that higher self-worth would provide some protection from the development of social anxiety symptoms. Given some similarity in the socioenvironmental predictors of self-worth and rejection sensitivity, as well as similar links with social anxiety, we considered global self-worth as a predictor (along with emotion dysregulation) to better isolate rejection sensitivity as a unique risk for the development of social anxiety symptoms during adolescence.

\section{Child Temperament and Social Anxiety}

Child temperamental traits that indicate greater inhibition are also well-established risk factors for social anxiety (La Greca \& Ranta, 2015; Muris \& Ollendick, 2005). In particular, shyness and behavioral inhibition, which are closely related temperamental traits defined as reticence and withdrawal from novel social and non-social situations, have been associated with heightened risk for social anxiety symptoms and disorder in childhood and adolescence (Spence $\&$ Rapee, 2016). For example, among a sample of adolescents, high levels of behavioral inhibition (comprised of infant behavioral observations and maternal reports of shyness) when reported multiple times from as early as 14 months of age was associated with higher parent- and self-reports of social anxiety symptoms and increased risk for a diagnosis of social anxiety 
disorder by adolescence (Chronis-Tuscano et al., 2009).

Negative affectivity is another often identified temperamental risk for anxious symptomology in youth. Negative affectivity involves general subjective distress, unpleasurable engagement with one's social environment, and negative mood states (Eisenberg et al., 2000). Numerous studies have reported that pre-adolescent negative affectivity increases the risk for many disorders including social anxiety (Eisenberg et al., 1998; Waters et al., 2012). Given this past evidence, these multiple indicators of temperamental traits were considered as potential predictors that would also uniquely account for adolescent social anxiety symptoms.

\section{The Current Study}

Theory and research on the development of anxiety during childhood and adolescence identify biases in the form of attentional allocation to threat (e.g., threat vigilance) and socialinformation processing biases (e.g., interpretation of ambiguous information) as risk factors for the development of anxiety. Yet, one cognitive-affective bias that involves expectations and emotional reactions to social information regarding acceptance and rejection (i.e., rejection sensitivity) has rarely been examined as an early risk factor for social anxiety symptom development during adolescence. Given the substantial evidence that fulfilling human needs for belongingness and acceptance are basic to human health and well-being (Baumeister \& Leary, 1995), rejection sensitivity would be expected to be a risk for many forms of maladjustment, but perhaps most especially social anxiety. The primary purpose of this 5-year longitudinal, multireporter study was to determine the role of rejection sensitivity in predicting growth in social anxiety symptoms over adolescence unique from other known or expected risk and protective factors for social anxiety, including peer rejection (peer-reported), emotion dysregulation, global self-worth, and temperament (parent-reported). Finally, we accounted for the impact of gender 
(La Greca \& Ranta, 2015; Masters et al., 2019; Nelemans et al., 2019) and tested if growth in social anxiety symptoms varied depending on the grade students were in at the first assessment.

\section{Method}

\section{Participants}

The participants were 377 boys (45\%) and girls (55\%) in grades $5(26 \%), 6(32 \%)$, or 7 (42\% overall participation rate) at $\mathrm{T} 1$ who participated in at least one wave of a 7 -wave longitudinal study conducted over five years. At study entry, students attended one of three participating schools in an urban area of Australia and ranged in age from 10 to 13 years $(M=$ 12.0, $S D=0.90$ ). The three schools (referred to as colleges in Australia) were comprised of both junior (preparatory year to grade 6) and senior (grade 7 to 12) schools that were in close

proximity to each other on the same campus. Each grade level in each school had approximately 80 to 120 students.

Most students endorsed white/Caucasian (79\%) or Asian (15\%) sociocultural background, with 1\% Australian First People / Torres Strait Islander / Pacific Islander, and the remaining 5\% endorsing "other." At the last wave (T7) of the study, students were in grades 10 to 12 and were age 15 to 18 years $(M=16.7, S D=0.86)$. Parents (almost always the mother, $n=$ $318,84 \%$ ) also completed a short survey during the consent process. Parents' mean age at T1 was 44.4 years $(S D=5.8$ years $)$. Most parents reported being married or living with a partner (85\%), with $14 \%$ reporting divorce or separation, and $1 \%$ single, never married. About $49 \%$ of parents had some university education, $21 \%$ reported no education beyond high school, and the remaining parents $(30 \%)$ reported some technical or other training beyond high school.

We considered publicly available school and regional demographic information to assess the representative of the participating students. It is important to note that questions regarding 
birth country and language spoken at home are often asked in Australia instead of the questions asked about race/ethnicity in this study. The schools from which the students were drawn report that their student population (all grades) is approximately 52\% boys, with $1 \%$ Australian first peoples or Pacific Islander, and about $20 \%$ speaking a language other than English at home. The schools report that $10 \%$ of students are in the lowest income quartile, $61 \%$ are in the middle two income quartiles, and 29\% are in the highest income quartile. A regional demographic survey reports $64 \%$ of adults born in Australia, 1.7\% First Peoples or Pacific Islander, 17\% with a university degree (18\% Year 12 high school maximum, 12\% Year 10 high school maximum, with $53 \%$ reporting some education beyond high school), and $45 \%$ married. Relative to the available school demographic information, our study participants had a slightly higher proportion of girls but was representative otherwise. In comparison to the region, the students in this study had more educated parents, which was likely also reflected in the distribution of income levels in the schools. Students had a higher proportion of married parents than in the adult population in the region, but this would likely be the result of families defined by having children.

\section{Procedure}

Study approval from the Griffith University Human Research Ethics Committee (Protocol \#2013/13) was obtained before school principals were contacted and parent/student consent were sought. Overall, $58 \%$ of consent forms were returned to the school, and there was a $42 \%$ participation rate. The return and consent rate may have been adversely affected by the request that parents complete a questionnaire. No student with parent consent declined W1 participation. A small gift (e.g., keyring) was given to students after each survey was completed.

Students completed the first four assessments six months apart. A planned missingness design (Little \& Rhumtella, 2013) was used so that 50\% of students completed the social anxiety 
measure items at wave 1 and the other $50 \%$ of students completed it at the next wave, alternating this for the first four waves. All students completed the social anxiety measure at wave 5 to wave 7. For the measure of rejection sensitivity, $50 \%$ of students completed it at wave 1 , and the other $50 \%$ completed it at wave 2 . All students completed the measure of general self-worth and ratings of peers at both wave 1 and wave 2 . At wave 1 , parents completed a survey to report child temperament, and emotion dysregulation was only collected at wave 3 . For this study, we collapsed the social anxiety data across wave 1 and wave 2 and across wave 3 and wave 4 . We also collapsed scores for rejection sensitivity, general self-worth and peer rejection across wave 1 and wave 2. Thus, a total of five waves of data were formed for the analyses reported here (wave $1 / 2$, wave $3 / 4$, wave 5 , wave 6 , and wave 7 ). To account for different timing of symptom measures, we created a dichotomous variable indicating whether students first completed social anxiety items at wave 1 or wave 2 and included this indicator as a control variable in the conditional growth curve models. This indicator was not associated with anxiety and did not change the study results substantially; thus, it was trimmed from the models reported here.

We refer to the five reconfigured waves of data as Time 1 (T1) to Time 5 (T5). The lag between waves was about one year. All T1, T2, and T3 assessments were conducted in students' regular classrooms. At T4 and T5, one school opted for students to complete questionnaires in their regular classrooms (as before), but other students were contacted individually and completed an online survey or completed the survey via mail.

\section{Measures}

Social anxiety symptoms. The 18-item Social Anxiety Scale for Adolescents (SAS-A; La Greca \& Lopez, 1998) assessed social anxiety symptoms at each wave of the study. Each item (e.g., "I worry what others say about me") has five response options, ranging from 1 (not true) to 
5 (very true). The SAS-A contains items that assess fear of negative evaluation, social avoidance and distress in new situations, and general social avoidance and distress. Items were averaged to form a total social anxiety score, with higher scores indicating more anxiety. Cronbach's $\alpha$ ranged from .92 to .95 , with Cronbach's $\alpha$ of .92 for the items merged across waves 1 and 2 , and .92 for waves 3 and 4 .

Anxious rejection sensitivity. Participants completed a short form of the Children's Rejection Sensitivity Questionnaire (CRSQ; Downey et al., 1998b). Six hypothetical rejection scenarios were used, with three related to peers and three related to teachers ("Imagine that a famous person is coming to visit your school. Your teacher is going to pick five kids to meet this person. You wonder if she will choose YOU”). Following each scenario, two questions were answered. The first two questions assessed anxiety (e.g., "How nervous would you feel about whether or not your teacher will choose you?') about the situation. The response options for these questions ranged from 1 (not at all) to 6 (extremely). The second question asked the participant to report their response expectation (e.g., do you think your teacher will choose you?). Response options to this question ranged from 1 (Yes!) to 6 (No!). rejection sensitivity anxious expectation scores for each scenario were calculated as the cross-product of the anxiety item and the response expectation item. The cross-products were then averaged to form a composite rejection sensitivity score, Cronbach's $\alpha=.76$ at wave 1 and .77 at wave 2 .

Cronbach's $\alpha$ was .76 for the merged wave 1 and 2 items.

General self-worth. Five items from the Self-Perception Profile for Adolescents (SPPA; Harter, 2012) measured self-worth. The SPPA employs an alternate choice format (e.g., "Some people are often disappointed with themselves" BUT "Other people are pretty pleased with themselves"). First, respondents decided which of the pair of statements best reflected them, and 
second, they decided if it was "really true" or "sort of true." Response options were re-scored to range from 1 (low esteem) to 4 (high esteem), and averaging items formed the total score. In the present study, Cronbach's $\alpha$ was .76 at wave 1 and .86 at wave 2 , and was .78 for the items averaged across the two waves.

Emotion dysregulation. The Difficulty in Emotion Regulation Scale (DERS; Gratz \& Roemer, 2004) measured emotion dysregulation (Neumann et al., 2010). Five subscales from the DERS were included: lack of emotional clarity, non-acceptance of emotional responses, impulse control difficulties, limited access to strategies, and difficulties engaging in goal-directed behavior. Response options ranged from 1 (never) to 5 (almost always). Some items required reverse coding prior to averaging to form subscales and then averaging to form a DERS composite score. Cronbach's $\alpha$ was .90 for all DERS items.

Peer report of peer rejection. The roster-rating method of liking was used (Asher \& Dodge, 1986; see also Bukowski et al., 2000). The method involved participants rating liking of each student in the classroom on a scale from 1 (not at all) to 5 (very much). To produce a score for each participant, ratings of 1 or 2 were treated as "not liked" and these received ratings were counted. A final proportional score to indicate peer rejection was formed for each participant by dividing the total number of 1 or 2 ratings by the total possible number of ratings. The possible range for these scores was 0 to $1 ; 96.3 \%$ of students received at least one rating of 1 or 2 and $83 \%$ of students had scores of .5 or less. Only one student received all ratings of 1 or 2 (i.e., a score of 1). The proportional rejection score was correlated with the (reversed) average of all ratings received, $r=.70, p<.001$. The rejection score was slightly more strongly correlated with all other measures when compared to the average of all ratings.

Parent report of adolescents' temperamental traits. Parents completed the 60 -item 
Early Adolescent Temperament Questionnaire (EATQ-R; Ellis \& Rothbart, 1999). The EATQ-R measures seven temperamental (activation control: 7 items, affiliation: 6 items, attention: 6 items, fear: 6 items, frustration: 6 items, high intensity pleasure: 9 items, inhibitory control: 5 items, and shyness: 5 items) and two behavioral scales (aggression: 6 items and depressive mood: 5 items). These can be combined into three global composites to indicate effortful control (attention, inhibitory control, activation control), surgency (high intensity pleasure, reversed fear, reversed shyness), and negative affect (frustration, depressive mood, aggression). Affiliation is maintained as a separate trait. Item response options ranged from 1 (almost always untrue of your child) to 5 (almost always true of your child). To produce global temperament trait sores, items were averaged within subscales and then averaged to form global composites. Cronbach's $\alpha$ was .89 for effortful control, .81 for surgency, .84 for negative affect, and .70 for affiliation.

\section{Overview of Analyses}

Overall, 33 students missed one (9\%), 19 students missed two (7.5\%), 11 students missed three $(3 \%)$, and 11 students missed four (3\%) of the waves of data collection. Also, $318(84 \%)$ parents completed the measure of temperament. Given there few participants had missing data at T1, most missing data were due to attrition at follow-up assessments (i.e., social anxiety measures) and parent-reported temperament. There were no missing data for peer ratings. Based on Little's (1988) MCAR test, student-reported data were missing completely at random (MCAR), $\chi^{2}=88.9, p=.101$, and Little's MCAR was also not significant, $\chi^{2}=201.5, p=.061$ when parent-reported data were included in the analysis. We also tested whether any measure under consideration in this study predicted overall missingness. T1 social anxiety and T1 parentreported effortful control, but no other measures, were significantly associated with the count of total missing scores, with adolescents higher in social anxiety, $r=.11(p=.036)$, and lower 
effortful control, $r=-.15(p=.011)$, missing more data. Considering these findings, we maintained all participants in this study by replacing missing data using multiple imputation (20 imputed datasets), and pooled means, $S D$ s, and correlations between all variables were reported. For the primary analyses, missing data were estimated with FIML while fitting growth curve models using AMOSv25. We fit unconditional and conditional latent-variable growth curve models of social anxiety symptoms, and model fits were assessed with commonly used indices, including the $\chi^{2}$-test and associated level of significance, and the Comparative Fit Index (CFI) (Bentler \& Bonett, 1980). The Root Mean Square Error of Approximation (RMSEA) (Browne \& Cudeck, 1993) gave an estimate of error due to approximate fit of the models.

First, unconditional latent growth curve models were estimated to identify the best fitting model of social anxiety symptom growth for all participants, and to test whether there was significant interindividual variability in intercepts and patterns of growth. Next, three conditional latent growth models were fit. The first model was constructed to test whether rejection sensitivity was predictive of the intercept and growth in social anxiety. In this model grade and gender were entered as additional predictors because $\mathrm{T} 1$ grade level ranged from 5 to 7 , which is a period where some grade- or age-related differences in social anxiety symptoms have been found (Ding et al., 2020), and gender differences in social anxiety symptom levels have been widely supported in past research (Bowker et al., 2011; Nelemans et al., 2019). In a second latent growth curve model, we added all time-invariant predictors (i.e., self-worth, emotion dysregulation, peer rejection, and temperamental traits) to determine if associations of rejection sensitivity with social anxiety intercept and growth parameters remained significant despite adjustment for multiple other, more widely studied, correlates of social anxiety patterns during adolescence. Third, a trimmed latent growth model was fit, removing all predictors that were not 
significantly associated with any social anxiety growth parameter.

\section{Results}

\section{Descriptive Statistics and Correlations between Measures}

Correlations between measures, and the means and SDs of measures are provided in Table 1 . Social anxiety was positively correlated with $\mathrm{T} 1$ rejection sensitivity, emotion dysregulation, and negative affectivity, and intermittently positively correlated with T1 peer rejection. Social anxiety was consistently negatively correlated with general self-worth, effortful control, and surgency. Most T1 measures were significantly intercorrelated with each other.

\section{Unconditional Growth Models}

To test unconditional growth in social anxiety, we fit a linear growth model, followed by fitting a second model to test for a quadratic growth pattern. In each model, the growth parameters were free to covary with each other (e.g., the intercept and the slope). In the linear growth model, social anxiety linearly increased from T1 to T5 $(B=.08, p<.001)$ and there was significant interindividual variability $(p<.001)$ in both the estimated intercepts and slopes of social anxiety. This model had an adequate fit to the data, $\chi 2(10)=54.55, p<.001, \mathrm{CFI}=.96$, RMSEA $=.109(.082-.138), p<.001$. The quadratic model also had a good fit, $\chi 2(6)=23.90, p$ $<.01, \mathrm{CFI}=.99, \mathrm{RMSEA}=.089(.054-.128), p=.037$, which was significantly better than the fit of the linear model, $\Delta \chi 2(4)=30.65, p<.01$. The quadratic term was significant $(B=.01, p=$ $.026)$, and the linear slope estimate was no longer significantly larger than $0(B=.02, p=.556)$. There was significant variability in the estimated intercepts $(p<.001)$, linear slopes $(p=.017)$ and quadratic growth $(p<.001)$ in social anxiety. Thus, we fit conditional growth curve models building on the quadratic latent growth model.

\section{Conditional Models of Social Anxiety Growth Patterns}


Gender, grade, and rejection sensitivity. The first conditional latent growth model estimated the effect of $\mathrm{T} 1$ rejection sensitivity on social anxiety symptoms, when considered alongside only gender and grade (see Table 2, Model 1). Rejection sensitivity, gender, and grade were freed to have directional effects on social anxiety intercept, slope, and quadratic terms, and the intercept, slope, and quadratic terms were freed to covary with each other. Also, rejection sensitivity, gender, and grade were first freed to covary with each other, but nonsignificant covariances were trimmed. This model had a very good fit to the data, $\chi 2(14)=37.02, p<.01$, CFI $=.98$, RMSEA $=.066(.041-.092), p=.138$. Rejection sensitivity was associated with a higher intercept and less positive slope in social anxiety from $\mathrm{T} 1$ to $\mathrm{T} 5$, and also was associated with a more prominent quadratic pattern of growth. Gender (girls higher than boys), but not grade, was associated with a higher social anxiety intercept (see Table 2). Grade also had a significant association with the quadratic pattern, with grade level associated with a lesser quadratic shape. The significant intercept, slope and quadratic variances indicated interindividual variation in social anxiety growth left to explain.

Full model: Gender, grade, rejection sensitivity, and all other predictors. The second conditional model built on the first by adding all additional measured predictors (see Table 2, Model 2). In this model, all predictors were first freed to covary with each other, but nonsignificant covariances were trimmed. This model had a very good fit to the data, $\chi 2(43)=$ $98.71 p<.01, \mathrm{CFI}=.97, \mathrm{RMSEA}=.059(.043-.074), p=.164$. Gender $($ girl $)$, rejection sensitivity, emotion dysregulation, and negative affectivity were each associated with a higher intercept of social anxiety. Surgency was associated with a lower intercept. Regarding predictors of the linear slope of social anxiety symptoms, rejection sensitivity was associated with a less positive slope, and emotion dysregulation was associated with a more positive slope. Three T1 
predictors were also associated with quadratic growth, with a positive association for rejection sensitivity and negative affectivity, and a negative association for emotion dysregulation.

Final model. A final conditional model was fit after trimming predictors that were not significantly associated with social anxiety intercept and growth patterns (see Table 2, Model 3). This model had a very good fit to the data, $\chi 2(19)=43.90, p<.01, \mathrm{CFI}=.99$, RMSEA $=.059$ $(.036-.082), p=.236$. The results were similar to those for the full model for gender, rejection sensitivity, and emotion dysregulation. However, trimming nonsignificant predictors slightly modified some of the associations of negative affectivity and surgency with social anxiety. Negative affectivity was negatively associated with the linear slope but positively associated with the quadratic growth pattern. Surgency was not associated with social anxiety intercept, slope or quadratic growth.

To depict the effect of rejection sensitivity on social anxiety patterns over time in this model, predicted growth patterns are shown in Figure 1 for adolescents low (-1 SD), average, and high (+1 SD) in T1 rejection sensitivity. As shown, the temporal pattern of social anxiety symptoms had a more pronounced concave shape at a high (+1 SD) level of rejection sensitivity relative to a low or average level. Similar illustrations of the effects of negative affectivity and emotion dysregulation are shown in Figures 2 and 3, respectively. As can be seen in Figure 2, patterns of social anxiety symptoms over time at low, average and high levels of negative affectivity were similar to those for rejection sensitivity. As can be seen in Figure 3, the temporal patterns of social anxiety symptoms followed a more pronounced concave pattern at a low level (-1 SD) of emotion dysregulation relative to average and high levels dysregulation.

\section{Follow-up Model of Rejection Sensitivity Anxiety and Expectations}

As a follow-up, we refit the final model including the anxious component of rejection 
sensitivity separate from the expectation component of rejection sensitivity. This model had an adequate fit to the data, $\chi 2(24)=103.03, p<.01, \mathrm{CFI}=.96, \mathrm{RMSEA}=.094(.075-.113), p<$ .001 . The rejection sensitivity components were each associated with a higher social anxiety intercept $(B=.201$ and .303 for anxiety and expectation, respectively, both $p<.001)$, but only rejection sensitivity expectation (not anxiety) was significantly associated with the linear slope $(B=-.132, p<.001)$ and with quadratic growth in social anxiety $(B=.021, p<.001)$.

\section{Discussion}

The pre-adolescent to early adolescent transitional period is crucial for the identification and prevention of several factors that can increase adolescents' vulnerability to social anxiety (Chronis-Tuscano et al., 2018; La Greca \& Ranta, 2015; Spence \& Rapee, 2016). Even if symptoms are transient and never rise to the level of seeking mental health care or a clinical diagnosis of disorder, higher levels of social fear and avoidance of negative evaluation in social situations can be disruptive to optimal development. Therefore, identifying factors that may help to prevent or protect against social anxiety symptoms in adolescence remains a central focus for developmental and clinical scientists. We investigated whether rejection sensitivity measured at the transition to adolescence, characterized by early occurring biases in expecting and misinterpreting cues of rejection, might be a catalyst for growth in social anxiety across adolescence, when considered alongside other known or expected risk and protective factors.

\section{Rejection Sensitivity and Social Anxiety Growth during Adolescence}

As expected, there were associations of early rejection sensitivity with the intercept of social anxiety symptoms, but also with the linear slope and quadratic (i.e., curvilinear) pattern of symptoms over the following five years of adolescence. Generally consistent with what was expected, adolescents highest in rejection sensitivity at the start of the study had the highest level 
of social anxiety symptoms. In addition, adolescents who reported more rejection sensitivity had a more pronounced quadratic (i.e., curvilinear) shape of symptoms over the following five years. However, somewhat unanticipated was the finding that youth higher in rejection sensitivity had less linear increase in anxiety so, conversely, adolescents with average and even low rejection sensitivity relative to their peers showed a pattern more consistent with linear increase. Although unanticipated, this finding is not an uncommon one when considering risk factors for changes in symptomatology over time; linear growth can be less pronounced among adolescents who initially report a high level of risk or have the most elevated symptom intercept (see ZimmerGembeck et al., 2018). Thus, overall, adolescents who report high rejection sensitivity at the transition to adolescence are higher in social anxiety symptoms from the first years of adolescence and their symptoms do not abate when assessed into later adolescence. Also noteworthy, adolescents with average, and even low, rejection sensitivity scores have a concerning pattern of growth in social anxiety symptoms as they move into and through middle adolescence.

Even when considered alongside other known or expected risk and protective factors for social anxiety, including peer rejection, emotion dysregulation, self-worth, and temperament (as well as female gender), rejection sensitivity conferred risk for social anxiety symptoms. These findings suggest that rejection sensitivity is a unique risk factor for a high level of social anxiety symptoms across the early years of adolescence and can identify youth at risk of continued symptoms into middle to later adolescence. In particular, and consistent with theory and research on the risk that social-information biases present in the development of youth anxiety (Spence \& Rapee, 2016), adolescents higher in rejection sensitivity are more likely to interpret neutral or ambiguous social cues as rejection, react strongly to overt experience of rejection, and become 
more distressed from these social interactions into the future (Downey \& Feldman, 1996; Gardner et al., 2020; Zimmer-Gembeck, 2016). These findings support the relevance for continued examination of rejection sensitivity as another cognitive-affective risk factor for maintenance of, or increases in, social anxiety symptoms. It is possible that adolescents higher in rejection sensitivity experience greater attentional allocation to threat within their social interactions, become more overwhelmed by such threat (Zimmer-Gembeck, 2016), all of which may place them at continued risk for social anxiety. It would be useful for future research to test these notions by focusing on identifying the more precise mechanisms by which rejection sensitivity confers risk for social anxiety disorder in later adolescence or adulthood.

\section{Other Findings}

Gender and grade level. Turning to other findings, consistent with previous studies and reviews on the developmental course of social anxiety (e.g., La Greca \& Ranta, 2015; Nelemans et al., 2019; Rapee et al., 2019), on average, social anxiety symptoms appeared stable in level in the early adolescent years, but then showed an upturn beginning at about age 14. Further, when gender and grade level were considered in the full model, gender was associated with the social anxiety intercept in the most comprehensive model (girls higher in social anxiety than boys), but grade level was not. These findings confirm past research identifying girls' greater vulnerability to social anxiety (La Greca \& Lopez, 1998; Nelemans et al., 2019), but linear or quadratic change in symptoms was not significantly predicted by gender.

Other measured risk factors for social anxiety growth. In addition to the key findings for rejection sensitivity, both emotion dysregulation and the temperamental trait of negative affectivity (as reported by parents) were associated with a higher intercept of social anxiety and related to growth in symptoms over adolescence in our most comprehensive models. Adolescents 
who reported a higher level of dysregulation in early adolescence (such as fewer strategies for managing emotions and more impulsive emotions) were more likely to follow a linear pattern of symptoms over time (thus, flatter quadratic growth in symptoms). Furthermore, adolescents who were reported to be higher in negative affectivity by their parents had more social anxiety symptoms and showed a sharper quadratic pattern of growth in social anxiety beginning in middle adolescence. Theory has identified emotion regulation deficits combined with high negative affectivity as a general risk for psychopathology (e.g., Thompson, 2019). Although this view suggests examining an interaction effect, which we did not do here given our focus on rejection sensitivity, our findings do support the importance of emotion dysregulation and negative affectivity in social anxiety symptom development during adolescence.

Although associated with a lower intercept of social anxiety in the full models, it was surprising that surgency, another temperamental traits considered as a risk for social anxiety, was not associated with social anxiety growth in the final trimmed model. Surgency comprised aspects of fear and shyness (albeit reversed and focused on lack of fear and shyness); fearfulness has been identified as a risk for heightened social anxiety (Chronis-Tuscano et al., 2018; Spence \& Rapee, 2016). This finding could be a result of tapping low shyness when measuring surgency. Studies of temperament in children often find that shyness is not always associated with social anxiety, providing a distinction between individuals who are highly inhibited (compared to stable patterns of inhibition) often preferring to be alone, demonstrating a non-fearful tendency to be alone (similar to preference for solitude) compared to the fearful anxiety and avoidance shown in social anxiety (Eisenberg et al., 1998; 2000). Previous studies also suggest that indices of effortful control, inhibition, and positive affectivity may interact with high emotionality or negative affectivity to predict social anxiety (Muris \& Ollendick, 2005; Rothbart et al., 2011; 
Rapee et al., 2019). Future research designed to identify a range of personal and social risks for social anxiety development would benefit from making finer distinctions between aspects of regulation, affectivity, fear, and shyness and include a measure that taps each aspect.

It is also noteworthy that general self-worth was not significantly associated with social anxiety growth in our final model. Nevertheless, general self-worth was correlated with fewer symptoms of social anxiety. Thus, consistent with previous findings (Bowker et al., 2011; Gómez-Ortiz et al., 2017), self-worth can be positive for well-being, but it was not a unique predictor of social anxiety growth during adolescence when rejection sensitivity, emotion dysregulation, and negative affectivity were simultaneously considered.

\section{Study Limitations, Future Research Directions and Conclusion}

Before concluding, there are four study limitations to mention. First, some measures were reported by parents and peers, but self-report was the predominant data collection method. We did this because self-report is often the most accurate when assessing symptoms or perceptions of others (e.g., Costello \& Angold, 1995). Nevertheless, reliance on self-report for multiple measures may have resulted in shared method variance, which has the potential to inflate associations. Second, about $16 \%$ of parents did not complete the measure of temperament and there were additional data missing when adolescents were not present at school or available for personal follow-up. We imputed missing data using best practices to maintain all participants in the study. However, the higher amount of missing parent-report data may have had some influence on the results. Third, while we addressed a significant gap by examining rejection sensitivity as a unique predictor of social anxiety growth, we did not examine change in predictors. Therefore, we did not examine how risk and protective factors may covary over time with social anxiety symptoms, and did not test whether social anxiety symptoms also convey risk 
for increased rejection sensitivity over time. Future research could address these limitations. A final limitation to mention is the grade range of adolescents at the start and throughout each wave. This range was somewhat wide, spanning three grades (and three ages). Future research could focus on a narrower age range or recruit a larger sample to allow for age-specific analyses. In summary, the average pattern of social anxiety symptoms found in this study was quadratic in shape, showing an increase starting when adolescents were, on average, 14 years of age. This quadratic pattern was shifted upwards towards higher symptoms in girls relative to boys. Regarding risk factors, as predicted, youth higher in rejection sensitivity at the transition to adolescence had more symptoms and were also at risk for continued or increased symptoms into later adolescence. Also, adolescents higher in emotion dysregulation and negativity affectivity at the start of the study were at greater risk for social anxiety symptom development. These findings suggest multiple risk factors for social anxiety. Studies of adolescents designed to identify additional biases and risk experiences associated with social anxiety would add to the findings presented here. Research that considers social anxiety disorder (rather than or in addition to symptoms), other aspects of temperament, and other forms of peer problems would be particularly useful. Also relevant, researchers could include measures of family risk factors (such as parent mental health disorders and overprotection or accommodation of anxious symptoms; Hudson \& Rapee, 2001; Waters et al., 2012) and biological and neuropsychological development (e.g., puberty; Guyer et al., 2016). Overall, however, we expect that expanding our models to include additional risk factors would not undermine the relevance of rejection sensitivity, emotion dysregulation, and temperamental negative affectivity in the development of social anxiety during adolescence. 


\section{References}

Abela, J. R. Z., \& Hankin, B. L. (2009). Cognitive vulnerability to depression in adolescents: A developmental psychopathology perspective. In S. Nolen-Hoeksema \& L. M. Hilt (Eds.), Handbook of depression in adolescents (pp. 335-376). Routledge.

Adrian, M., Jenness, J. L., Kuehn, K. S., Smith, M. R., \& McLaughlin, K. A. (2019). Emotion regulation processes linking peer victimization to anxiety and depression symptoms in adolescence. Development and Psychopathology, 31(3), 999-1009. https://doi.org/10.1017/S0954579419000543.

Asher, S. R., \& Dodge, K. A. (1986). Identifying children who are rejected by their peers. Developmental Psychology, 22(4), 444-449. https://doi.org/10.1037/0012-1649.22.4.444

Ayduk, O., Downey, G., Testa, A., Yen, Y., Shoda, Y. (1999). Does rejection elicit hostility in rejection sensitive women? Social Cognition, 17(2), 245-271. https://doi.org/10.1521/soco.1999.17.2.245

Baumeister, R. F., \& Leary, M. R. (1995). The need to belong: Desire for interpersonal attachments as a fundamental human motivation. Psychological Bulletin, 117(3), 497-529.

Bentler, P. M., \& Bonett, D. G. (1980). Significance tests and goodness-of-fit in the analysis of covariance structures. Psychological Bulletin, 88(3), 588-606. https://doi.org/10.1037/0033-2909.88.3.588.

Bowker, J. C., Thomas, K. K., Norman, K. E., \& Spencer, S. V. (2011). Mutual best friendship involvement, best friends' rejection sensitivity, and psychological maladaptation. Journal of Youth and Adolescence, 40(5), 545-555. https://doi.org/10.1007/s10964-010-9582-x.

Bowlby, J. (1969). Attachment and loss: Vol. 1. Hogarth.

Browne, M. W., \& Cudeck, R. (1993). Alternative ways of assessing model fit. In K. A. Bollen 
\& J. S. Long (Eds.), Testing structural equation models (pp. 136-162). Sage.

Bukowski, W. M., Sippola, L., Hoza, B., \& Newcomb, A. F. (2000). Pages from a sociometric notebook: An analysis of nomination and rating scale measures of acceptance, rejection, and social preference. New Directions in Child and Adolescent Development, 88, 11-26. https://doi.org/10.1002/cd.23220008804.

Carthy, T., Horesh, N., Apter, A., \& Gross, J. J. (2010). Patterns of emotional reactivity and regulation in children with anxiety disorders. Journal of Psychopathology and Behavioral Assessment, 32(1), 23-36. https://doi.org/10.1007/s10862-009-9167-8.

Chango, J. M., McElhaney, K., Allen, J. P., Schad, M. M., \&Marston, E. (2012). Relational stressors and depressive symptoms in late adolescence: Rejection sensitivity as a vulnerability. Journal of Abnormal Child Psychology, 40(3), 369-379. https://doi.org/10.1007/s10802-011-9570-y.

Chronis-Tuscano, A., Danko, C. M. Rubin, K. H., Coplan, R. J. \& Novick, D. R. (2018). Future directions for research on early intervention for young children at risk for social anxiety. Journal of Clinical Child \& Adolescent Psychology, 47(4), 655-667. https://doi.org/10.1080/15374416.2018.1426006.

Chronis-Tuscano, A., Degnan, K. A., Pine, D. S., Perez-Edgar, K., Henderson, H. A., Diaz, Y...Fox, N. A. (2009). Stable early maternal report of behavioral inhibition predicts lifetime social anxiety disorder in adolescence. Journal of American Academy of Child and Adolescent Psychiatry, 48(9), 928-935. https://doi.org/10.1097/CHI.0b013e3181ae09df.

Clear, S. J., Gardner, A. A., Webb, H. J., \& Zimmer-Gembeck, M. J. (2020). Common and distinct correlates of depression, anxiety and aggression: Attachment and emotion regulation of sadness and anger. Journal of Adult Development, 27, 181-191. 
https://doi.org/10.1007/s10804-019-09333-0

Compas, B. E., Jaser, S. S., Bettis, A. H., Watson, K. H., Gruhn, M. A., Dunbar, J. P.,...Thigpen, J. C. (2017). Coping, emotion regulation, and psychopathology in childhood and adolescence: A meta-analysis and narrative review. Psychological Bulletin, 143(9), 939991. https://doi.org/10.1037/bul0000110.

Costello, E. J., \& Angold, A. (1995). Developmental epidemiology. Epidemiologic Reviews, 17(1), 74-82. https://doi.org/10.1093/oxfordjournals.epirev.a036187

Ding, X., Fu, R., Ooi, L. L., Coplan, R. J., Zheng, Q., \& Deng, X. (2020). Relations between different components of rejection sensitivity and adjustment in Chinese children. Journal of Applied Developmental Psychology, 67(Mar-Apr), 101119. https://doi.org/10.1016/j.appdev.2020.101119

Downey, G., \& Feldman, S. I. (1996). Implications of rejection sensitivity for intimate relationships. Journal of Personality and Social Psychology, 70(6), 1327-1343. https://doi.org/10.1037/0022-3514.70.6.1327.

Downey, G., Freitas, A. L., Michaelis, B., \& Khouri, H. (1998a). The self-fulfilling prophecy in close relationships: Rejection sensitivity and rejection by romantic partners. Journal of Personality and Social Psychology, 75(2), 545-560. https://doi.org/10.1037//00223514.75.2.545.

Downey, G., \& Bonica, C. \& Rincón, C. (1999). Rejection sensitivity and adolescent romantic relationships. In W. Furman, Brown, B. B., \& Feiring, C. (Eds.), The development of romantic relationships (pp. 148-174). Cambridge University Press.

Downey, G., Lebolt, A., Rincón, C., \& Freitas, A. L. (1998b). Rejection sensitivity and children's interpersonal difficulties. Child Development, 69(4), 1074-1091. 
https://doi.org/10.1111/j.1467-8624.1998.tb06161.x

Dryman, M. T., \& Heimberg, R. G. (2018). Emotion regulation in social anxiety and depression: A systematic review of expressive suppression and cognitive reappraisal. Clinical Psychology Review, 65 (Nov), 17-42. https://doi.org/10.1016/j.cpr.2018.07.004.

Duffy, A. L., Gardner, A. A., \& Zimmer-Gembeck, M. J. (2020). Peer rejection and dislike. In M. J. Zimmer-Gembeck \& A. M. Waters (Volume Editors), S. Hupp \& J. D. Jewel (Series Eds.). The Encyclopedia of Child and Adolescent Development: Emotion in Childhood (pp. 1727-1740). Wiley. https://doi.org/10.1002/9781119171492.wecad191.

Eisenberg, N., Fabes, R. A., Guthrie, I. K., \& Reiser, M. (2000). Dispositional emotionality and regulation: Their role in predicting quality of social functioning. Journal of Personality and Social Psychology, 78(1), 136-157. https://doi.org/10.1037//0022-3514.78.1.136.

Eisenberg, N., Shepard, S. A., Fabes, R. A., Murphy, B. C., \& Guthrie, I. K. (1998). Shyness and children's emotionality, regulation, and coping: contemporaneous, longitudinal, and across-context relations. Child Development, 69(3), 767-790. https://doi.org/10.1111/j.1467-8624.1998.tb06242.x.

Ellis, L. K., \& Rothbart, M. K. (1999). Early Adolescent Temperament Questionnaire-Revised. University of Oregon.

Gardner, A. A., Zimmer-Gembeck, M. J., \& Modecki, K. (2020). A longitudinal model of rejection sensitivity and internalizing symptoms: Testing emotion regulation deficits as a mechanism and outcome of symptoms. Journal of Personality. https://doi.org/10.1111/jopy.12549.

Gardner, A., \& Zimmer-Gembeck, M. J. (2018). Rejection sensitivity and responses to rejection: Serial mediators linking parenting to emerging adults' depression and trait anxiety. Journal 
of Relationships Research, e9. https://doi.org/10.1017/jrr.2018.8

Golombek, K., Lidle, L., Tuschen-Caffier, B., Schmitz, J., \& Vierrath, V. (2019). The role of emotion regulation in socially anxious children and adolescents: A systematic review. European Child \& Adolescent Psychiatry. https://doi.org/10.1007/s00787-019-01359-9.

Gómez-Ortiz, O., Roldán, R., Ortega-Ruiz, R., \& García-López, L. (2017). Social anxiety and psychosocial adjustment in adolescents: Relation with peer victimization, self-esteem and emotion regulation. Child Indicators Research. https://doi.org/10.1007/s12187-017-9506-3

Gratz, K. L., \& Roemer, L. (2004). Multidimensional assessment of emotion regulation and dysregulation: Development, factor structure, and initial validation of the Difficulties in Emotion Regulation Scale. Journal of Psychopathology and Behavioral Assessment, 26(1), 41-54. https://doi.org/10.1023/B:JOBA.0000007455.08539.94.

Gross, J. J., \& Jazaieri, H. (2014). Emotion, emotion regulation and psychopathology: An affective science perspective. Clinical Psychological Science, 2(4), 387-401. https://doi.org/10.1177/2167702614536164.

Guyer, A. E., Silk, J. S., \& Nelson, E. E. (2016). The neurobiology of the emotional adolescent: From the inside out. Neuroscience and Biobehavioral Reviews, 70(Nov), 74-86. https://doi.org/10.1016/j.neubiorev.2016.07.037

Hankin, B. L., \& Abramson, L. Y. (2001). Development of gender differences in depression: An elaborated cognitive vulnerability-transactional stress theory. Psychological Bulletin, 127(6), 773-796. https://doi.org/10.1037/0033-2909.127.6.773

Harter, S. (2012). The self-perception profile for adolescents. University of Denver.

Hudson, J. L., \& Rapee, R. M. (2001). Parent-child interactions and anxiety disorders: An observational study. Behaviour Research and Therapy, 39(12), 1411-1427. 
https://doi.org/10.1016/S0005-7967(00)00107-8.

La Greca, A. M., \& Lopez, N. (1998). Social anxiety among adolescents: Linkages with peer relations and friendships. Journal of Abnormal Child Psychology, 26(2), 83-94. https://doi.org/10.1023/A:1022684520514.

La Greca, A. M., \& Ranta, K. (2015). Developmental transitions in adolescence and their implications for social anxiety. In K. Ranta, A. M. La Greca, L. Garcia-Lopez \& M. Marttunen (Eds.), Social Anxiety and Phobia in Adolescents: Development, Manifestation and Intervention Strategies (pp. 95-117). Springer

Laursen, B., \& Collins, A. W. (2009). Parent-adolescent relationships and influences. In R. M. Lerner, \& Steinberg, L. (Ed.), Handbook of adolescent psychology: Contextual influences on adolescent development (3rd ed., pp. 3-42). Wiley.

Little, R. J. A. (1988). A test of missing completely at random for multivariate data with missing values. Journal of the American Statistical Association, 83(404), 1198-1202. https://doi.org/10.1080/01621459.1988.10478722

Little, T. D., \& Rhemtulla, M. (2013). Planned missing data designs for developmental researchers. Child Development Perspectives, 7(4), 199-204. https://doi.org/10.1111/cdep.12043

Lopez, C., \& DuBois, D. L. (2010). Peer victimization and rejection: Investigation of an integrative model of effects on emotional, behavioral, and academic adjustment in early adolescence. Journal of Clinical Child and Adolescent Psychology, 34(1), 25-36. https://doi.org/10.1207/s15374424jccp3401_3

Masters, M. R., Zimmer-Gembeck, M. J., \& Farrell, L. J. (2019). Transactional associations between adolescents' emotion dysregulation and symptoms of social anxiety and 
depression: A longitudinal study. Journal of Early Adolescence, 39(Oct), 1085-1109. https://doi.org/10.1177/0272431618806053

Mathews, B. L., Kerns, K. A., \& Ciesla, J. A. (2014). Specificity of emotion regulation difficulties related to anxiety in early adolescence. Journal of Adolescence, 37(Oct), 10891091. https://doi.org/10.1016/j.adolescence.2014.08.002

Muris, P., \& Ollendick, T. H. (2005). The role of temperament in the etiology of child psychopathology. Clinical Child and Family Psychology Review, 8 (Dec), 271-289. https://doi.org/10.1007/s10567-005-8809-y

Nelemans, S. A., Meeus, W. H. J., Branje, S. J. T., Van Leeuwen, K., Colpin, H., Verschueren, K., \& Goossens, L. (2019). Social anxiety scale for adolescents (SAS-A) short form: Longitudinal measurement invariance in two community samples of youth. Assessment, 26(2), 235-248. https://doi.org/10.1177/1073191116685808

Neumann, A., Van Lier, P. A. C., Gratz, K. L., \& Koot, H. M. (2010). Multidimensional assessment of emotion regulation difficulties in adolescents using the difficulties in emotion regulation scale. Assessment, 17(1), 138-149. https://doi.org/10.1177/1073191109349579

Rapee, R. M., Oar, E. L., Johnco, C. J., Forbes, M. K., Fardouly, J., Magson, N. R., \& Richardson, C. E. (2019). Adolescent development and risk for the onset of socialemotional disorders: A review and conceptual model. Behaviour Research and Therapy. https://doi.org/10.1016/j.brat.2019.103501

Rothbart, M. K., Ellis, L. K., \& Posner, M. I. (2011). Temperament and self-regulation. In K. D. Vohs \& R. F. Baumeister (Eds.), Handbook of self-regulation: Research, theory, and applications (p. 441-460). Guilford Press. 
Rowe, S., Zimmer-Gembeck, M. J, Rudolph, J., \& Nesdale, D. (2015). A longitudinal study of rejecting and autonomy-relevant parenting, rejection sensitivity, and socioemotional symptoms in early adolescents, Journal of Abnormal Child Psychology, 43(6), 1107-1118. https://doi.org/10.1007/s10802-014-9966-6

Sackl-Pammer, P., Jahn, R., Özlü-Erkilic, Z., Pollak, E., Ohmann, S., Schwarzenberg, J...Akkaya-Kalayci, T. (2019). Social anxiety disorder and emotion regulation problems in adolescence. Child and Adolescent Psychiatry and Mental Health, 13, 37-49. Article number 37. https://doi.org/10.1186/s13034-019-0297-9.

Spence, S. H., \& Rapee, R. M. (2016) The etiology of social anxiety disorder: An evidencebased model. Behaviour Research and Therapy, 86(Nov), 50-67. https://doi.org/10.1016/j.brat.2016.06.007

Suveg, C., \& Zeman, J. (2004). Emotion regulation in children with anxiety disorders. Journal of Clinical Child and Adolescent Psychology, 33(4), 750-759. https://doi.org/10.1207/s15374424jccp3304_10

Thompson, R. A. (2019). Emotion dysregulation: A theme in search of definition. Development and Psychopathology, 31(3), 805-815. https://doi.org/10.1017S0954579419000282.

Troop-Gordon, W. (2017). Peer victimization in adolescence: The nature, progression, and consequences of being bullied within a developmental context. Journal of Adolescence, 55 (Feb), 116-128. https://doi.org/10.1016/j.adolescence.2016.12.012

Waters, A. M., Zimmer-Gembeck, M. J. \& Farrell, L. J. (2012). The relationships of child and parent factors with anxiety symptoms in children? Parental anxious rearing as a mediator. Journal of Anxiety Disorders, 26(7), 737-745. https://doi.org/10.1016/j.janxdis.2012.06.002 
Weeks, M., Coplan, R. J., \& Kingsbury, A. (2009). The correlates and consequences of early appearing social anxiety in young children. Journal of Anxiety Disorders, 23(7), 965-972. https://doi.org/10.1016/j.janxdis.2009.06.006.

Williams, K. D. (2001). Ostracism: The power of silence. New York: Guilford.

Zimmer-Gembeck, M. J. (2016). Peer rejection, victimization, and relational self-system processes in adolescence: Toward a transactional model of stress, coping, and developing sensitivities. Child Development Perspectives, 10(2), 122-127. https://doi.org/10.1111/cdep.12174.

Zimmer-Gembeck, M. J., Nesdale, D., McGregor, L., Mastro, S., Goodwin, B., \& Downey, G. (2013). Comparing reports of peer rejection: Associations with rejection sensitivity, victimization, aggression, and friendship, Journal of Adolescence, 36(6), 1237-1246. https://doi.org/10.1016/j.adolescence.2013.10.002

Zimmer-Gembeck, M. J., Trevaskis, S, Nesdale, D, \& Downey, G. (2014). Relational victimization, loneliness and depressive symptoms: Indirect associations via self and peer reports of rejection sensitivity, Journal of Youth and Adolescence, 43(4), 568-582. https://doi.org/10.1007/s10964-013-9993-6.

Zimmer-Gembeck, M. J., Webb, H. J., Farrell, L. J., \& Waters, A. M. (2018). Girls' and boys' trajectories of appearance anxiety from age 10 to 15 years are associated with earlier maturation and appearance-related teasing. Development and Psychopathology, 30(1), 337350. https://doi.org/10.1017/S0954579417000657. 
Table 1

Means and Standard Deviations (SDs) of Measures and Correlations between Measures $(N=377)$

\begin{tabular}{|c|c|c|c|c|c|c|c|c|c|c|c|c|c|c|}
\hline & & 1 & 2 & 3 & 4 & 5 & 6 & 7 & 8 & 9 & 10 & 11 & 12 & 13 \\
\hline 1 & Social anxiety & -- & & & & & & & & & & & & \\
\hline 3 & T3 social anxiety & $.57 * *$ & $.77 * *$ & -- & & & & & & & & & & \\
\hline 4 & T4 social anxiety & $.51 * *$ & $.61 * *$ & $.65^{* *}$ & -- & & & & & & & & & \\
\hline 5 & T5 social anxiety & $.52 * *$ & $.61 * *$ & $.63 * *$ & $.79 * *$ & -- & & & & & & & & \\
\hline 6 & Rejection sensitivity & $.63^{* *}$ & $.52 * *$ & $.47^{* *}$ & $.38^{* *}$ & $.39 * *$ & -- & & & & & & & \\
\hline 7 & Self-worth & $-.40 * *$ & $-.42 * *$ & $-.35^{* *}$ & $-.30 * *$ & $-.33 * *$ & $-.49 * *$ & -- & & & & & & \\
\hline 8 & Emotion dysregulation & $.46^{* *}$ & $.57 * *$ & $.60 * *$ & $.42 * *$ & $.40 * *$ & $.43^{* *}$ & $-.42 * *$ & -- & & & & & \\
\hline 9 & Peer-report rejection & $.17 * *$ & .09 & $.11 *$ & .08 & $.12 *$ & $.21 * *$ & $-.18 * *$ & .13 & -- & & & & \\
\hline 11 & Effortful control & $-.15^{* *}$ & $-.14 * *$ & -.09 & -.08 & $-.15 * *$ & $-.23 * *$ & $.29 * *$ & $-.21 * *$ & $-.26 * *$ & $.17 * *$ & -- & & \\
\hline 12 & Surgency & -.09 & -.09 & -.09 & $-.12 *$ & $-.19 * *$ & -.09 & .07 & -.09 & $-.13^{*}$ & $.21 * *$ & $.14 * *$ & -- & \\
\hline 13 & Negative affectivity & $.27 * *$ & $.22 * *$ & $.20 * *$ & $.21 * *$ & $.25^{* *}$ & $.22 * *$ & $-.31 * *$ & $.16^{* *}$ & $.24 * *$ & $-.32 * *$ & $-.44 * *$ & $-.13 *$ & -- \\
\hline & Mean & 2.22 & 2.20 & 2.25 & 2.48 & 2.53 & 9.19 & 3.26 & 2.09 & 0.32 & 3.96 & 3.39 & 3.58 & 2.50 \\
\hline & $S D$ & 0.83 & 0.78 & 0.84 & 0.91 & 0.96 & 4.62 & 0.71 & 0.63 & 0.19 & 0.46 & 0.50 & 0.52 & 0.43 \\
\hline
\end{tabular}

$* p<.05 . * * p<.01$

Note. All measures were completed at Time 1 (T1) except where indicated with Time 2 (T2) to Time 5 (T5). The possible range for all measures was 1 to 5 , with the exceptions of peer-report rejection, rejection sensitivity and self-worth. Peer-report rejection range was 0 to 1 . Rejection sensitivity possible range was 1 to 36 . Self-worth possible range was 1 to 4 . 
Table 2

Associations of Gender, Grade, Rejection Sensitivity, Self-worth, Emotion Dysregulation, Peer Rejection, and Temperamental Traits with Patterns of Growth in Social Anxiety across Adolescence

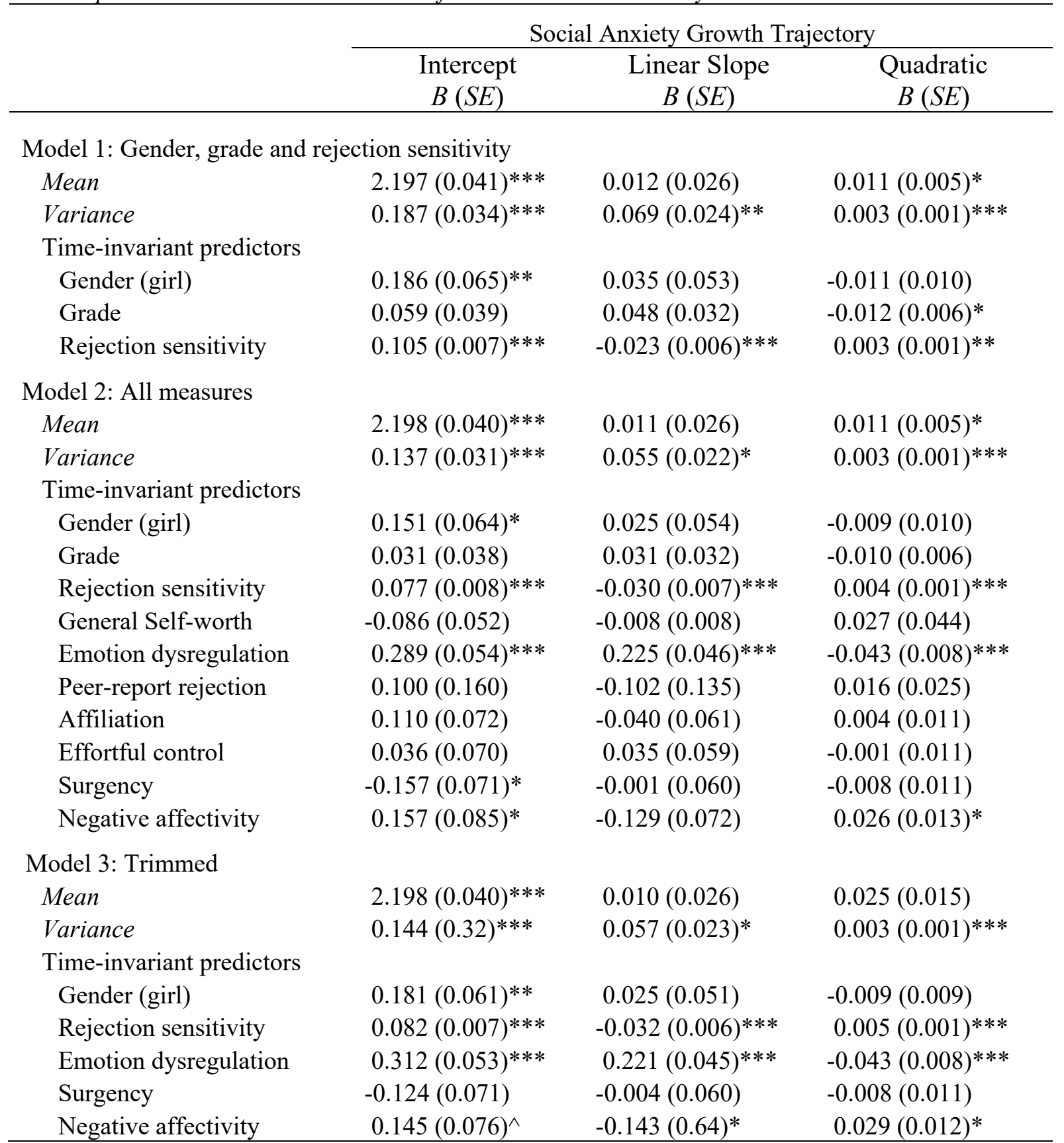

${ }^{*} p<.05 .{ }^{* *} p<.01 .{ }^{* * *} p<.001 .{ }^{\wedge} p=.058$

Note. Unstandardized coefficients are shown.

Model 1: $\chi 2(14)=37.02, p<.01, \mathrm{CFI}=.98, \mathrm{RMSEA}=.066(.041-.092), p=.138$.

Model 2: $\chi 2(43)=98.71, p<.01, \mathrm{CFI}=.97, \mathrm{RMSEA}=.059(.043-.074), p=.164$.

Model 3: $\chi 2(19)=43.90, p<.01, \mathrm{CFI}=.99, \mathrm{RMSEA}=.059(.036-.082), p=.236$. 


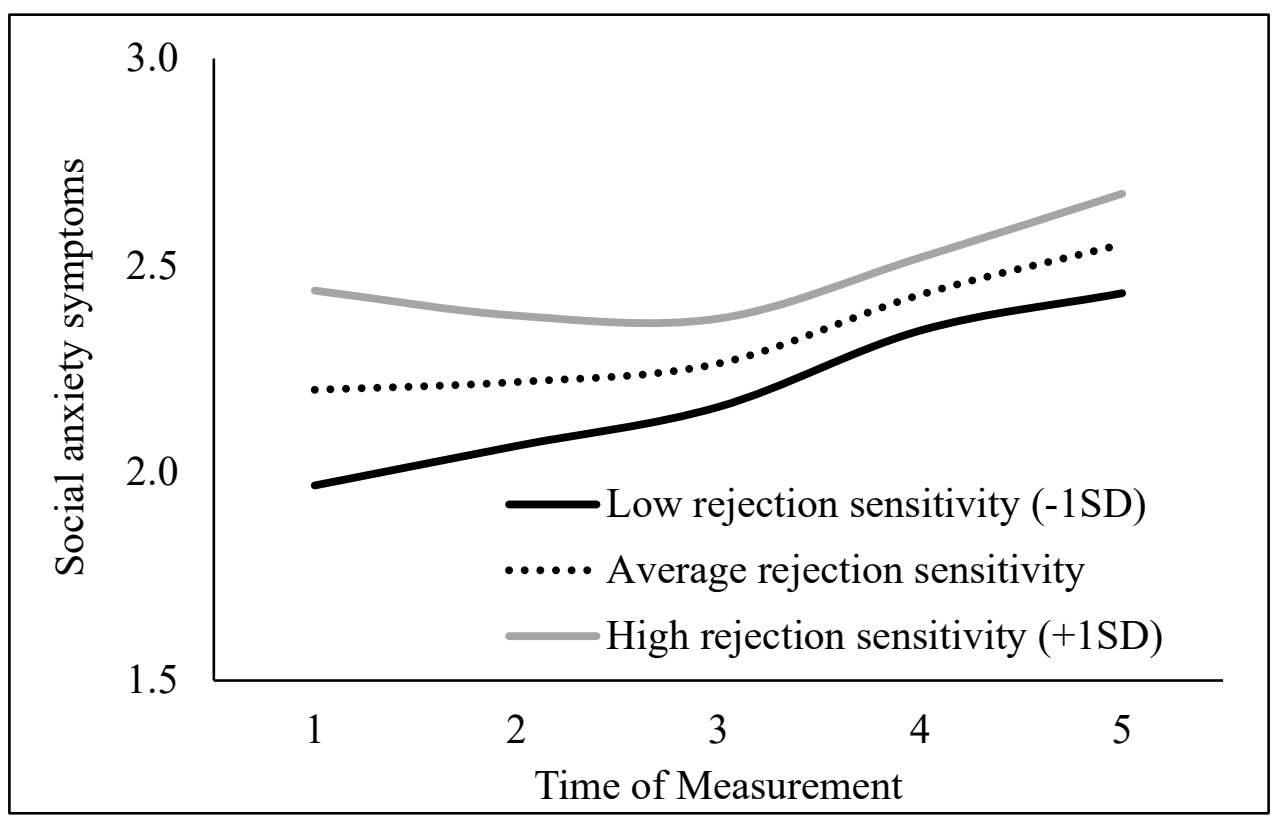

Figure 1. Illustration of predicted growth patterns of social anxiety symptoms for adolescents with low (1SD), average, and high (+1SD) T1 rejection sensitivity scores.

Note. There was an approximate 1-year lag between each time of measurement. Social anxiety scores can range from 1 to 5. Other predictors in the model (gender, negative affectivity, emotion dysregulation, and surgency) were set to the mean. 


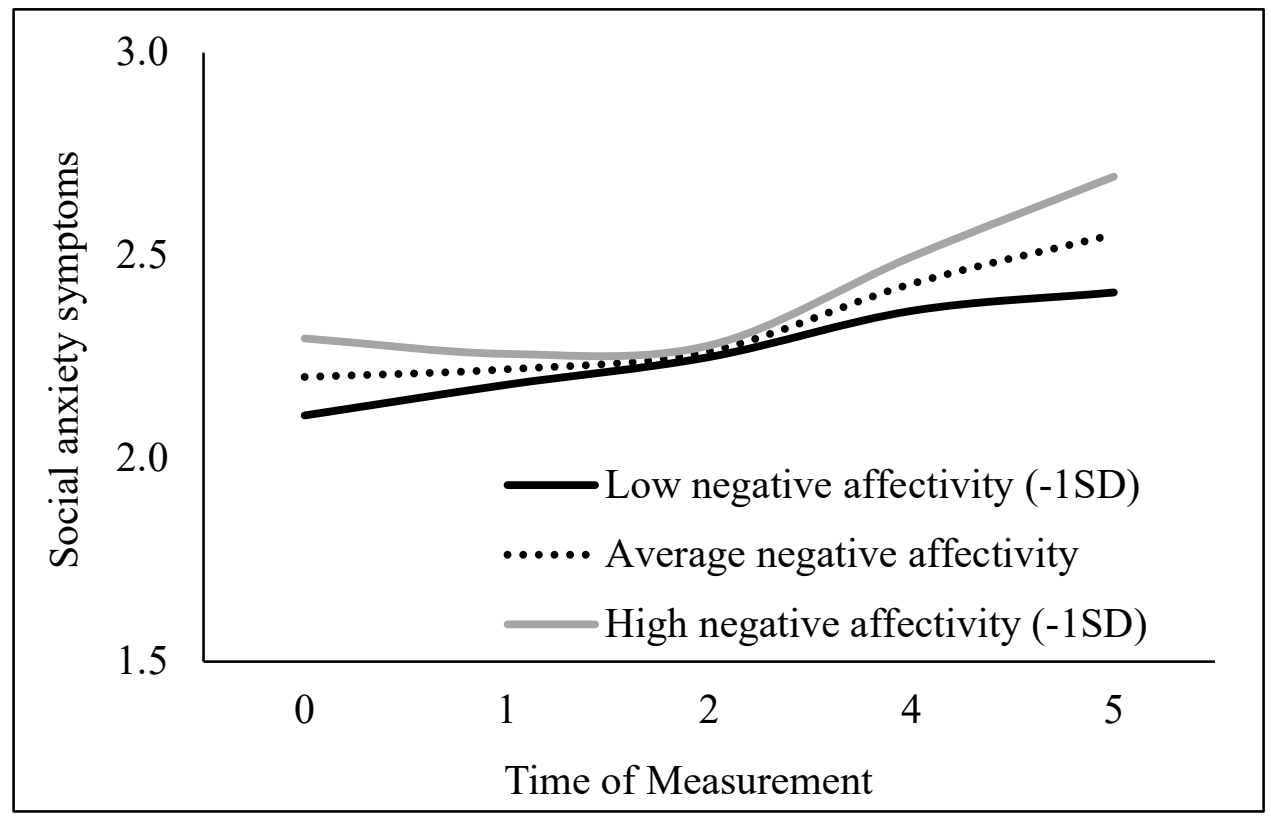

Figure 2. Illustration of predicted growth patterns of social anxiety symptoms for adolescents with low (1SD), average, and high (+1SD) T1 negative affectivity scores.

Note. There was an approximate 1-year lag between each time of measurement. Social anxiety scores can range from 1 to 5. Other predictors in the model (gender, rejection sensitivity, emotion dysregulation, and surgency) were set to the mean. 


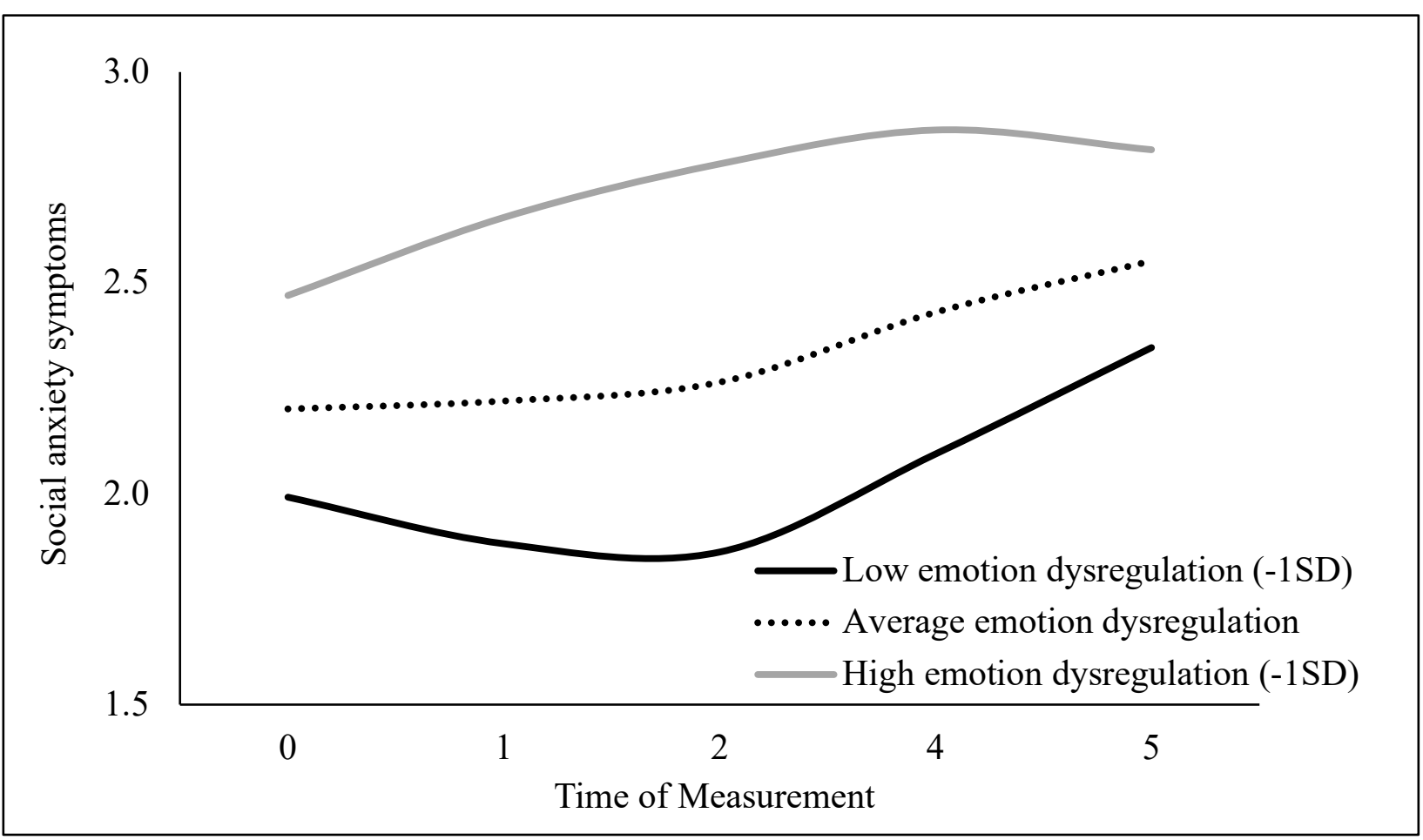

Figure 3. Illustration of predicted growth patterns of social anxiety symptoms for adolescents with low (1SD), average, and high (+1SD) T1 emotion dysregulation.

Note. There was an approximate 1-year lag between each time of measurement. Social anxiety scores can range from 1 to 5. Other predictors in the model (gender, rejection sensitivity, negative affectivity, and surgency) were set to the mean. 